\title{
L'ARBITRATO DEGLI AGENTI SPORTIVI DAVANTI AL COLLEGIO DI GARANZIA DELLO SPORT DEL CONI
}

\author{
Carlo Rasia \\ Professore associato di Diritto processuale civile nell'Università di Bologna. Docente del Laboratorio di \\ Arbitrato nello sport
}

\begin{abstract}
Il presente contributo intende analizzare, in occasione del suo recentissimo avvento, il fenomeno dell'“arbitrato degli agenti sportivi" istituito presso il CONI, evidenziandone tratti distintivi ed elementi di criticità, muovendo da due lodi arbitrali che hanno posto in rilievo una questione, della natura intrinsecamente processuale, relativa al termine decadenziale per la proposizione della domanda d'arbitrato.
\end{abstract}

Parole chiave: Giustizia sportiva, Arbitrato degli agenti sportivi, Regolamento degli agenti sportivi, Termine per la domanda d'arbitrato.

\begin{abstract}
The main purpose of this paper is to analyze the phenomenon of "Arbitrations of sports Agents", set up within the CONI, showing its features and critical elements, starting from two arbitral awards that have raised a question, of an intrinsically procedural nature, relating to the term for the submission of the request for arbitration.
\end{abstract}

Keywords: Sport justice, Arbitration of sports agents, Regulation of sports agents, Term for submitting the request for arbitration.

\section{I diversi compiti del Collegio di Garanzia}

Il Collegio di Garanzia dello Sport istituito presso il CONI costituisce l'organo di giustizia sportiva di ultima istanza cui è demandato il controllo di legittimità delle decisioni assunte in ambito federale una volta esperiti i gradi di giustizia interna. ${ }^{1}$

La decisione federale può, infatti, essere censurata, ai sensi dell'art. 54, comma 1, CGS avanti al Collegio "per violazione di norme di diritto, nonché per omessa o insufficiente motivazione circa un punto decisivo della controversia che abbia formato oggetto di disputa tra le parti'. Sulle orme del giudizio di cassazione, ${ }^{2}$ l'intento

\footnotetext{
${ }^{1}$ E. Lubrano, Il giusto processo sportivo innanzi il Collegio di Garanzia dello Sport, in Riv. dir. sportivo, 2020, p. 465 ss., sottolinea che "la scelta del Legislatore Sportivo appare ragionevole e proporzionata, nel senso di volere limitare l'ultimo grado di Giustizia Sportiva ad un controllo di mera legittimità, escludendo che si possa configurare un "terzo grado" di Giustizia Sportiva di merito (al pari dei due gradi di Giustizia Federale), come si era, invece, verificato nel precedente sistema con il TNAS, la cui cognizione estesa al merito aveva determinato, in tante occasioni, una riduzione consistente delle sanzioni disciplinari irrogate dalla Giustizia Federale (soprattutto nell'ambito del calcio) e dato luogo a non pochi malumori nei rapporti tra Giustizia Federale e Giustizia-CONI, nonché tra le stesse Federazioni ed il CONI. La scelta del Legislatore Sportivo, effettivamente, si è posta in linea con il ruolo del CONI nei confronti delle Federazioni Sportive, alla luce dei poteri di controllo del primo sulle seconde, come previsto dall'art. 7, comma 1, nonché dall'art. 23 dello Statuto del CONI". In questo senso, anche Collegio di Garanzia, sez. I, 13 febbraio 2019, n. 14.

2 M. Farina, Il Collegio di Garanzia dello Sport: competenze e procedimenti. Note a prima lettura, in Riv. dir. sportivo, 2015 , p. 114 ss., spec. p. 118, sottolinea che la limitazione del sindacato del Collegio di Garanzia alla violazione delle norme di diritto deve essere
} 
della norma è affidare a tale organo una funzione di legittimità a tutela dell'intero sistema sportivo, grazie al tramite di un sindacato a critica vincolata, ove risulta inammissibile un ricorso che prospetta censure differenti da quelle aventi ad oggetto uno dei su indicati motivi.

A tale Collegio, tuttavia, vengono attribuite, quale organo di merito di primo e unico grado, anche le controversie ad esso devolute "dalle altre disposizioni del presente codice", "da delibere della giunta nazionale del CONI", nonché "dagli Statuti e dai Regolamenti federali sulla base di speciali regole procedurali definite d'intesa con il CONI". 3

Proprio l'art. 54, comma 3, CGS getta, pertanto, le basi per l'istituzione di un arbitrato in seno al Collegio di Garanzia, attraverso la predisposizione di apposite procedure con annessi regolamenti che le disciplinano. A tale fine, il legislatore sportivo ha individuato quattro ruoli arbitrali svolti dal Collegio di Garanzia. Il primo concerne le procedure arbitrali tra Federazioni Sportive Nazionali, Discipline Sportive Associate ed Enti di Promozione Sportiva. In tali ipotesi, il Collegio di Garanzia esplica la funzione di disciplinare, attraverso un arbitrato di tipo irrituale e secondo equità, modelli di convenzioni normalmente redatti dal CONI. ${ }^{4}$ Un secondo procedimento riguarda 1'arbitrato in materia di Licenze UEFA; ${ }^{5}$ un terzo, già in atto nelle recenti stagioni 2019 e 2020, concerne le liti insorte in occasione all'adozione di provvedimenti di annullamento, prosecuzione e conclusione delle competizioni tra campionati, siano essi afferenti al settore professionistico o dilettantistico (art. 218 d.1. 19 maggio 2020, n. 34, convertito in 1.17 luglio 2020, n. 77). ${ }^{6}$ Quarta ed ultima figura di arbitrato rinvenibile in seno al Collegio di Garanzia è quella deputata alla risoluzione delle liti tra gli agenti sportivi e i loro assistiti. ${ }^{7}$

È di questa, nota quale "arbitrato degli agenti sportivi", che ci occuperemo in questo contributo.

\section{L'arbitrato degli agenti sportivi istituito presso il CONI}

Va subito detto che l'arbitrato degli agenti sportivi ha trovato innanzitutto la propria legittimazione nel Regolamento CONI degli agenti sportivi ${ }^{8}$, da ultimo modificato il 20 luglio 2021 col quale è stato, tra l'altro, inserito l'istituto della domiciliazione in Italia per gli agenti sportivi UE ed extra-UE. ${ }^{9}$

congruamente rapportata all'opportunità di escludere che dinanzi all'organo di vertice della giustizia sportiva possa svolgersi un rinnovato esame di merito della controversia in vista di una diversa ricostruzione della quaestio facti ormai saldamente definita dalla sentenza impugnata.

${ }^{3}$ Trattasi di una competenza dal carattere eccezionale, come chiarito dallo stesso Collegio di Garanzia, sez. I, 1 ottobre 2019, n. 77: "Le considerazioni svolte in merito al carattere eccezionale e residuale della competenza in grado unico e di merito riservata al Collegio di Garanzia dall'art. 54, comma 3, CGS CONI, sono state confermate anche dalle Sezioni Unite di questo Collegio che, pur consapevoli dei diversi orientamenti adottati nel tempo dalle singole Sezioni, hanno definitivamente chiarito che, fuori dai casi in cui prevale la tutela dell'ordine sportivo nazionale e dell'ordine pubblico complessivamente considerato, "la corretta interpretazione delle citate disposizioni dell'art. 54 del CGS del CONI consentano al Collegio di Garanzia di decidere in unico grado solo nei casi in cui ciò sia stato espressamente previsto. Con la conseguente necessità di assicurare, nell'ambito della giustizia federale, la prima ordinaria tutela delle posizioni soggettive che si ritengano lese per atti e provvedimenti adottati dagli organi federali” (così Coll. Gar., Sez. Un., decisione n. 62 del 7-11 settembre 2018)".

${ }^{4}$ Approvato con Deliberazione n. 1622 del Consiglio Nazionale CONI del 18 dicembre 2018. Come previsto dall'art. 1 del regolamento "Il Collegio di Garanzia dello Sport presso il CONI (di seguito, per brevità, Collegio di Garanzia), ai sensi dell'art. 54, comma 3, del Codice di Giustizia Sportiva, è l'Organo Arbitrale per le controversie tra Federazioni Sportive Nazionali, Discipline Sportive Associate ed Enti di Promozione Sportiva relative al "Modello di Convenzione" reso disponibile dal CONI.

${ }^{5}$ Approvato con Deliberazione n. 1550 del Consiglio Nazionale CONI del 4 maggio 2016. Il Collegio di Garanzia è l'organo arbitrale cui, per l'art. 1 del regolamento, "sono devolute le controversie tra la FIGC e una società cui sia stata negata o revocata la Licenza UEFA a seguito della pronuncia della Commissione di secondo grado delle Licenze UEFA istituita presso la FIGC".

${ }^{6}$ Approvato con deliberazione del Presidente del CONI n. 38/23 del 10 giugno 2020. All'art. 1 si stabilisce: "Il presente Regolamento stabilisce le norme che regolano il procedimento innanzi al Collegio di Garanzia dello Sport per la risoluzione delle controversie di cui all'art. 218 del decreto legge 19 maggio 2020, n. 34, aventi ad oggetto i provvedimenti relativi all'annullamento, alla prosecuzione e alla conclusione delle competizioni e dei campionati, professionistici e dilettantistici, ivi compresa la definizione delle classifiche finali, per la stagione sportiva 2019/2020, nonché i conseguenti provvedimenti relativi all'organizzazione, alla composizione e alle modalità di svolgimento delle competizioni e dei campionati professionistici e dilettantistici, per la successiva stagione sportiva 2020/2021".

${ }^{7}$ Il Regolamento è stato approvato con deliberazione del Consiglio Nazionale CONI n. 1654 del 17 dicembre 2019.

${ }^{8}$ Approvato con deliberazione n. 1630 del Consiglio Nazionale del 26 febbraio 2019.

${ }^{9}$ Il Regolamento CONI agenti sportivi nonché del relativo Regolamento disciplinare è stato aggiornato con delibera del 20 luglio 2021. Sono state apportate alcune modifiche alla normativa specialistica di settore, in particolare, per gli agenti registrati dopo il $1^{\circ}$ aprile 
Proprio tale Regolamento, all'art. 22, commi 1 e 2, affida al Collegio di Garanzia la competenza a giudicare, ai sensi dell'art. 54, comma 3, CGS, in sede giurisdizionale, i "ricorsi avverso i provvedimenti disciplinari adottati dalla Commissione CONI agenti sportivi" e, salvo espressa deroga contenuta nel contratto di mandato, in sede arbitrale, "tutte le controversie aventi ad oggetto la validità, l'interpretazione e l'esecuzione dei contratti di mandato stipulati dagli agenti sportivi, nonché le relative controversie di carattere economico".

Con questo duplice obiettivo, di giudice disciplinare da un lato, ${ }^{10} \mathrm{e}$ di giudice del merito contrattuale dall'altro, il Collegio di Garanzia decide a tutto tondo sui rapporti tra agenti sportivi e suoi assistiti.

Occupiamoci in questa sede di questo secondo profilo, analizzando come il Collegio di Garanzia risolve le liti economiche.

In forza dell'art. 22, comma 2, del Regolamento degli agenti, il legislatore ha deciso di far decidere le controversie economiche tra agenti e loro assistiti, salvo che le parti avessero diversamente stabilito, "al Collegio di Garanzia dello Sport di cui all'art. 12-bis dello Statuto del CONI, in funzione di arbitrato irrituale", emettendo a tal fine un Regolamento arbitrale a dicembre 2019.

Tale Regolamento, all'art. 1, recita che "il Collegio di Garanzia dello Sport presso il CONI, ai sensi dell'art. 54, comma 3, del Codice di Giustizia Sportiva, è l'organo arbitrale per le controversie aventi ad oggetto la validità, l'interpretazione e l'esecuzione dei contratti stipulati dagli agenti sportivi, nonché le relative controversie di carattere economico, ai sensi dell'art. 22, comma 2, del Regolamento degli agenti sportivi”. Il comma $2^{\circ}$, del medesimo articolo, aggiunge a sua volta che "ogni controversia che tragga origine ai sensi dell'art. 11, comma 2, del Regolamento CONI degli agenti sportivi, che preveda l'arbitrato libero o irrituale presso il Collegio di Garanzia, ad istanza di una o tutte le parti interessate, sarà risolta mediante arbitrato irrituale di equità da espletarsi secondo il presente Regolamento". ${ }^{11}$

Alla luce di tale norma bisogna chiedersi innanzitutto se, stipulato il contratto di mandato tra agente sportivo e assistito, sia obbligatoria la devoluzione delle liti all'arbitrato del Collegio di Garanzia.

Va detto che il predetto contratto di mandato disciplinato dal regolamento prevede di default una clausola che impone di compromettere le liti in arbitri avanti al Collegio, clausola operante salvo che le parti non ne facciano espressa deroga. Resta salva, pertanto, una possibilità di "opt out" e, quindi, di "uscita" dal campo arbitrale del Collegio di Garanzia, a tutela della natura volontaria e non anche vincolante del rimedio in questione che altrimenti lo renderebbe obbligatorio e dunque incostituzionale. ${ }^{12}$ Beninteso, la clausola compromissoria contenuta nel contratto di mandato è valida e non vessatoria ai sensi dell'art. 1341 c.c., non solo perché non si tratta di contratto predisposto unilateralmente da una delle parti, ma più in generale perché il rapporto negoziale intercorrente deve soddisfare le prescrizioni di forma e di sostanza stabilite dalla normativa federale, e dunque non

2015 presso Federazioni estere (UE ed Extra-UE) che intendano operare in Italia ricorrendo all'istituto della domiciliazione (la cui iscrizione si puntualizza sarà anch'essa per l'anno solare) sin dalla sessione di calciomercato estiva 2021 anche svolgendo l'attività attraverso persona giuridica.

${ }^{10}$ Il Collegio di Garanzia è dunque anche organo di terza istanza competente a giudicare degli illeciti disciplinari commessi dagli agenti sportivi, dopo che la lite è stata decisa dalla Commissione federale agenti sportivi della FSN (in primo grado) e dalla Commissione agenti sportivi CONI (in grado di appello). La materia è disciplinata dal Regolamento disciplinare agenti sportivi CONI, approvato con deliberazione della Giunta Nazionale n. 128 del 14 maggio 2020.

11 Sulla ratio della scelta in favore della natura irrituale dell'arbitrato sportivo, si segnala il principio di diritto già consolidato in giurisprudenza, ex multis, Cass., 28 agosto 2005, n. 18919, in Rivista di diritto ed economia dello sport, 2005, p. 195 ss., secondo cui devono configurarsi, in termini di clausole compromissorie per arbitrato irrituale, "le clausole contenute in contesti statutari e di regolamenti associativi con cui sia imposto ai soggetti partecipanti di deferire ad organi od autorità interne la risoluzione della controversia".

12 A tal riguardo, G. Napolitano, Caratteri e prospettive dell'arbitrato amministrato sportivo, in Giorn. Dir. amm., 2004, p. 1159 ss., laddove si evince che "bisogna distinguere tra arbitrati da legge e arbitrati obbligatori. Questi ultimi sono incostituzionali perché precludono, contro la volontà dell'interessato, il diritto di agire in giudizio per la tutela dei propri diritti. I primi, invece, sono ammissibili, perché la legge o altra fonte normativa si limita a predisporre l'istituto arbitrale e a sottoporlo a una decisione speciale. Le parti, però, rimangono libere di decidere se ricorrere all'istituto arbitrale, eventualmente anche assumendo ex ante un obbligo contrattuale in tal senso". 
è frutto di una libera determinazione delle parti. ${ }^{13} \mathrm{~A}$ ciò si aggiunga che al contratto di mandato non si deve nemmeno applicare l'asimmetria informativa che giustifica l'applicazione della normativa del Codice del Consumo. ${ }^{14}$ In altri termini, si può dire che la devoluzione delle liti relative al contratto di mandato tra agente ed assistito alla cognizione del Collegio di Garanzia ha fonte non già propriamente nel suddetto contratto, ma nella normativa che regolamenta la figura dell'agente e l'esercizio della sua attività.

In secondo luogo, un'ulteriore problematica riguarda gli arbitri e la loro nomina.

Va preliminarmente precisato che l'arbitrato di cui si parla è un arbitrato semi-amministrato dal Collegio di Garanzia dello Sport presso il CONI, ove quest'ultimo assume il ruolo di vera e propria Camera arbitrale. Qui i membri del Collegio di Garanzia si tolgono le vesti di componenti del Collegio e indossano quelle di componenti del collegio arbitrale. Peraltro, la stessa sede dell'arbitrato si trova presso gli uffici di Roma del Collegio di Garanzia e la segreteria del CONI svolge le medesime funzioni per il Collegio arbitrale.

Che vi sia una sovrapposizione di ruoli, è evidente anche dalla circostanza per cui tutti i provvedimenti, ivi inclusa la fissazione dei termini ritenuti opportuni per assicurare il tempestivo e regolare svolgimento dell'arbitrato fino alla data della nomina dell'arbitro unico o del Presidente, "sono assunti dal Presidente del Collegio di Garanzia".

Trattasi di un arbitrato irrituale le cui controversie sono decise secondo equità da arbitri unici o collegi arbitrali composti da tre arbitri, tutti tratti dall'elenco dei componenti del Collegio di Garanzia. In mancanza di scelta, si applica la collegialità. È data facoltà, ai sensi dell'art. 2 del regolamento, alla parte che adisce il Collegio nella sua funzione arbitrale di scegliere chi debba rivestire il ruolo di arbitro di parte, provvedendovi, solo in mancanza di scelta espressa, il Presidente del Collegio di Garanzia, cui spetta anche la nomina con decreto del Presidente del collegio.

Con questo particolare meccanismo, si realizza peraltro una parziale coincidenza tra l'organo di designazione e l'arbitro: dunque tra colui che nomina (il Presidente del Collegio di Garanzia) e colui viene nominato (il membro del Collegio di Garanzia). Tale soluzione può, in realtà, delineare potenziali conflitti di interesse che i Regolamenti di arbitrato amministrato hanno sempre impedito sul nascere, evitando commistioni di ruoli all'interno della Camere tra gli arbitri e i soggetti designatori. ${ }^{15}$ Inoltre, in un contesto in cui gli arbitri possono essere nominati solo tra i membri del Collegio di Garanzia, potrebbe verosimilmente verificarsi che una parte nomini sempre lo stesso arbitro. A tale proposito, le IBA Guidelines on conflict of interest invitano le parti ad evitare le nomine ripetute imponendo, attraverso la collocazione in una lista arancione di situazioni sintomatiche, agli arbitri di segnalare di essere già stati nominati in precedenza da una parte, così lasciando all'altra l'onere di decidere se accettare o meno tale condizione. ${ }^{16}$ Ritengo che tale circostanza possa costituire motivo di ricusazione dell'arbitro (ai sensi dell'art. 2, comma 7), nonché essere oggetto di un "duty of disclosure", nonostante quest'ultimo istituto non venga espressamente previsto dal testo regolamentare. ${ }^{17}$

\footnotetext{
${ }^{13}$ L. Santoro, Brevi note in tema di applicabilità della normativa sul contratto di consumo al mandato tra agente sportivo e assistito, in Riv. dir. economia trasporti e ambiente, 2020, p. 81 ss., affronta il tema sull'asserita vessatorietà della clausola compromissoria contenuta nei contratti di mandato per violazione dell'art. 33, lett. t) del d. lgs. n. 205/2006 ovvero per violazione dell'art. 1341, comma 2, c.c., in quanto non soggetta a specifica approvazione per iscritto. Sul punto anche il Lodo n. 1 del 2021, il quale evidenzia che il contratto tra agente e atleta "deve rispettare le prescrizioni di forma e di sostanza stabilite dalla normativa federale, nonché, a seguito dell'entrata in vigore della disciplina sulla professione di Agente Sportivo di cui all'art. 1, comma 373, della 1. n. $205 / 2017$ (Legge di Stabilità 2018), anche quella di fonte statale ivi contenuta e la normativa CONI che ne è seguita (Regolamento degli Agenti Sportivi e Regolamento arbitrale per la risoluzione delle controversie ex articolo 22, comma 2, Regolamento CONI Agenti Sportivi)".

${ }^{14}$ Nello stesso senso il Lodo arbitrale n.1 del 2021, in quanto l'atleta (in specie, il calciatore) non riveste la qualifica di consumatore.

${ }^{15}$ Vedi, in tal senso, quanto stabilisce l'art. 18 della Camera arbitrale di Milano che impedisce sia ai membri del Consiglio arbitrale (quelli che provvedono alle nomine) sia ai dipendenti della CAM di essere nominati quali arbitri.

${ }^{16}$ Le IBA Guidelines on Conflicts of Interest in International Arbitration così espressamente sanciscono: "The Orange List is a nonexhaustive list of specific situations that, depending on the facts of a given case, may, in the eyes of the parties, give rise to doubts as to the arbitrator's impartiality or independence [...] In all these situations, the parties are deemed to have accepted the arbitrator if, after disclosure, no timely objection is made".

${ }^{17}$ Nonostante trovi applicazione nei più importanti Regolamenti di arbitrato amministrato italiani e non solo.
} 
Venendo, ora, a taluni aspetti tipicamente processuali di tale arbitrato, va detto che le regole applicabili al procedimento sono quelle stabilite dal regolamento e, in via sussidiaria, dagli arbitri purché nel rispetto del contraddittorio (art. 9, comma 8). Non si fa alcun riferimento a fonti esterne, nemmeno di stampo statale, che tuttavia devono ritenersi comunque applicabili in via secondaria.

L'instaurazione dell'arbitrato ha luogo, ai sensi dell'art. 3, con istanza scritta, inviata a mezzo posta elettronica certificata, al Collegio di Garanzia, assieme al deposito del contributo amministrativo, ${ }^{18}$ entro il termine perentorio di venti giorni "dalla violazione contestata", con il quale, oltre alle deduzioni di merito e istruttorie, si provvede contestualmente alla nomina dell'arbitro designato. Fa seguito la notifica a cura dell'istante della relativa istanza alla parte intimata.

Parte intimata provvede, ai sensi dell'art. 4, alla nomina dell'arbitro entro i tre giorni successivi alla notifica della domanda d'arbitrato. Nei dieci giorni successivi alla notifica della domanda di arbitrato, parte intimata deposita presso la Segreteria del Collegio di Garanzia e notifica a parte istante la sua memoria difensiva, ben potendo l'istante eventualmente replicare alla domanda riconvenzionale nel termine di cinque giorni decorrenti a far data dalla notifica della memoria.

Entro tre giorni dal deposito della memoria di parte intimata, debitamente costituita, il Presidente del Collegio di Garanzia, raccogliendo l'accettazione della designazione dei due componenti e del Presidente dell'organo arbitrale, dà notizia dell'istaurazione del procedimento mediante comunicazione sul sito internet del CONI.

Nella prima udienza fissata, entro dieci giorni dall'ultima accettazione della nomina, si procede, ai sensi dell'art. 5, quale condizione di procedibilità, al tentativo di conciliazione: se la conciliazione è raggiunta si dà atto della conclusione dell'accordo nel verbale d'udienza ovvero in un separato documento allegato al verbale, diversamente, in caso di esito negativo del tentativo di conciliazione, il giudizio prosegue nelle forme ordinarie. Ad ogni modo, nulla osta, durante il corso della procedura, che il tentativo venga rinnovato.

Qualora l'Organo arbitrale, come previsto dall'art. 6, ritenga la causa matura per la decisione, fissa, nel termine più breve possibile, l'udienza nella quale le parti possono oralmente tenere le loro difese. Se, invece, la natura della controversia lo richieda, il collegio o l'arbitro unico può concedere i termini per lo scambio delle memorie difensive e repliche.

Potrebbero, d'altra parte, essere formulate istanze istruttorie: l'organo arbitrale, ai sensi dell'art. 7, ove ritenga necessaria l'istruttoria, ammette o, addirittura, dispone d'ufficio, i mezzi istruttori rilevanti nella stessa udienza ovvero si riserva di provvedere con separata ordinanza.

A differenza di quanto accade nell'arbitrato di diritto comune ex artt. 806 ss. c.p.c., l'arbitro è titolare di poteri coercitivi e di imperio, ben potendo provvedere all'adozione di misure cautelari: difatti, dispone l'art. 8 del Regolamento, che "possono essere richieste misure cautelari quando sussista un pericolo di danno grave ed irreparabile e ad un sommario esame sia possibile una ragionevole previsione dell'esito favorevole della lite". Vengono, in altri termini, riproposti, in sede di arbitrato degli agenti sportivi, il fumus bonis iuris e il periculum in mora quali presupposti per richiedere e ottenere la tutela cautelare. ${ }^{19}$

${ }^{18}$ Previsto a pena di procedibilità. L'art. 10 del Regolamento, ai commi 3 e 4 prevede: "3. In caso di mancata corresponsione dell'importo di cui al precedente comma 1 e di cui all'articolo 9, comma 6, ove richiesto, l'organo Arbitrale, previa diffida ad adempiere con un termine non inferiore a dieci giorni, sospende il procedimento, dandone comunicazione al Presidente del collegio e alle parti. La sospensione produce effetti anche sul termine di pronuncia del lodo di cui all'art. 9, comma 1, del presente Regolamento". "4. La sospensione è revocata dall'Organo Arbitrale, verificato l'adempimento. Decorsi sessanta giorni dalla comunicazione del provvedimento di sospensione senza che il versamento sia stato eseguito, il Presidente del collegio dichiara l'estinzione del procedimento, dandone comunicazione alle parti e all'Organo Arbitrale, rimettendo a quest'ultimo il provvedimento di liquidazione dell'attività".

${ }^{19}$ La norma riprende in toto l'art. 23, comma 1, del Codice dei giudizi innanzi al TNAS, organo ormai abrogato, che in passato prevedeva anche l'emanazione di una misura cautelare anche in assenza di contraddittorio e prima ancora che si fosse costituito il collegio arbitrale. Sulla precedente figura, cfr. P. Sandulli, L'arbitrato nel sistema di giustizia sportiva, in Aa. Vv., Sull'arbitrato. Studi offerti a Giovanni Verde, Jovene, Napoli, 2010, p. 768. Va aggiunto che la c.d. legge delega al Governo "per l'efficienza del processo civile e per la revisione della disciplina degli strumenti di risoluzione alternativa delle controversie e misure urgenti di razionalizzazione dei procedimenti in materia di diritti delle persone e delle famiglie nonché in materia di esecuzione forzata" (già approvata a novembre 2021 in via definitiva dai due rami del Parlamento e, al momento in cui si scrive, non ancora pubblicata) ha stabilito, tra i tanti, 
Conclusa l'udienza, ai sensi dell'art. 9, il Collegio arbitrale, già riunitosi in camera di consiglio, pronuncia il dispositivo della decisione, riservando la pubblicazione delle motivazioni nei quindici giorni successivi. Il termine per la conclusione del procedimento è comunque fissato in sessanta giorni dalla data di costituzione dell'Organo arbitrale e coincide con la data di deposito del lodo presso la segreteria del Collegio di Garanzia. Il termine di deliberazione del lodo può essere prorogato solo per accordo delle parti, non essendo prevista alcuna proroga da parte degli arbitri né ex lege. Il lodo è, però, ai sensi dell'art. 9, comma 3, inimpugnabile e vincola pienamente le parti già a far data della sua sottoscrizione da parte della maggioranza degli arbitri.

È doveroso comprendere il significato dell'impugnabilità del lodo e per farlo bisogna analizzare innanzitutto qual è la natura del provvedimento così pronunciato.

In linea generale, tutti i provvedimenti del Collegio di Garanzia sono atti amministrativi e non giurisdizionali, impugnabili, dunque, avanti al giudice amministrativo, quali atti del CONI. ${ }^{20}$ Tale circostanza non è priva di risvolti pratici e finisce per avere inevitabili implicazioni sul diritto ad impugnare della parte soccombente per vizi propri dell'atto amministrativo. A mio parere, alla medesima conclusione non può giungersi per quanto riguarda le decisioni arbitrali del medesimo collegio, decisioni che ritengo comunque impugnabili avanti al giudice ordinario.

A tale proposito, anche recente giurisprudenza amministrativa si è pronunciata in occasione di un'impugnazione di un lodo arbitrale irrituale pronunciato del Collegio di Garanzia. ${ }^{21}$ In quel caso, il TAR Lazio ha dichiarato inammissibile il ricorso per difetto di giurisdizione, ritenendo non rientrante la controversia "tra quelle per le quali sussiste la giurisdizione esclusiva del giudice amministrativo", in quanto avente ad oggetto diritti soggettivi di natura patrimoniale derivanti da contratti stipulati tra l'agente sportivo e un suo assistito. Viene, a tal fine, richiamato in motivazione il disposto normativo di cui all'art. 133, lett. z) c.p.a. che circoscrive la giurisdizione esclusiva del giudice amministrativo "alle controversie aventi ad oggetto atti del Comitato Olimpico Nazionale o delle Federazioni sportive non riservate agli organi di giustizia dell'ordinamento sportivo ed escluse quelle inerenti ai rapporti patrimoniali tra società, associazioni e atleti”. Orbene, il TAR non ha escluso l'impugnabilità del lodo irrituale pronunciato dal Collegio di Garanzia, ma ha negato la propria giurisdizione in favore di quella del giudice ordinario, correttamente confutando la natura amministrativa del provvedimento arbitrale del Collegio.

Tale tesi è condivisibile e non mi sembra che possa ritenersi allora impediente la rinuncia preventiva all'impugnazione stabilita dal regolamento, la quale non ha efficacia, al pari di quanto espressamente previsto per l'arbitrato rituale nell'art. 829 , comma 1, c.p.c.

Pertanto, sembra potersi concludere che la natura arbitrale (anche irrituale) della decisione del Collegio di Garanzia in tale materia non impedisce di per sé la sua impugnazione, stante la possibilità di sottoporre tale decisione, al pari di ogni determinazione contrattuale, al sindacato del giudice ordinario, quello del luogo di pronuncia del lodo. La competenza spetterà in questo caso al Tribunale in funzione di giudice del lavoro, poiché

l'obiettivo di riconoscere agli arbitri il potere di emanare provvedimenti cautelari. La legee delega, però, non si è spinta oltre il confine tracciato dall'arbitrato rituale. Un atteggiamento certamente più cauto se messo a confronto con i poteri di imperio riconosciuti, fin dall'emanazione del regolamento, agli arbitri irrituali del Collegio di Garanzia. V., per un maggiore approfondimento degli aspetti salienti della riforma in atto, C. Rasia, Commento. Note minime sulla proposta di legge delega in materia di arbitrato, in Giurisprudenza arbitrale, 2021, p. 182 ss.

${ }^{20}$ Il Collegio di Garanzia dello Sport si pone quale ultimo grado di giustizia sportiva con decisioni di natura amministrativa che possono essere oggetto di impugnazione o di contestazione innanzi al giudice amministrativo che è competente in maniera esclusiva ex art. 133, lett. z) c.p.a. In questo senso, E. Zucconi Galli Fonseca- C. Rasia, Laboratorio di arbitrato nello sport, Bononia University Press, Bologna, 2021, p. 18 ss. Recente giurisprudenza (Tar Lazio, sez. I-ter, 5 luglio 2021, n. 7937, in www.giustizia-amminsitrativa.it) ha stabilito che il Collegio di Garanzia dello Sport non ha personalità giuridica autonoma e distinta da quella del CONI ed emette atti a natura amministrativa e non giurisdizionale, sicché la legittimazione processuale va riconosciuta in capo al CONI. Atteso che le decisioni adottate da parte del Collegio di Garanzia incidono sull'oggetto della controversia, potendo modificare - in funzione nomofilattica - i provvedimenti sanzionatori adottati da parte delle singole Federazioni sportive, ai sensi dell'art. 12-bis comma 3 dello Statuto del CONI, sono proprio dette decisioni a dover essere contestate, se del caso, avanti al giudice amministrativo, con conseguente legittimazione passiva proprio del CONI.

${ }^{21}$ Il riferimento è alla sentenza del TAR Lazio, sez. I-ter, 7 ottobre 2021, n. 10246, in www.giustizia-amministrativa.it. 
trattasi di rapporti d'agenzia che, ex art. 409, comma 1, n. 3, c.p.c., rientrano nell'ambito della competenza di quest'ultimo. L'impugnazione potrà esser fatta valere per i motivi previsti dall'art. 808-ter c.p.c. e, in generale, per i motivi di nullità del contratto. ${ }^{22}$

\section{Il Lodo n. 1/2021 (conforme al precedente n. 6/2020) e il temine di decadenza della domanda arbitrale}

Particolare attenzione merita il già menzionato disposto normativo contenuto nell'art. 3 del Regolamento arbitrale, stante il quale "la procedura arbitrale è introdotta entro il termine perentorio di venti giorni dalla violazione contestata con istanza introdotta al Collegio di Garanzia...". La norma in esame fissa un termine perentorio, a pena di inammissibilità, a decorrere dalla "violazione contestata" per la proposizione della domanda arbitrale.

Ci si è interrogati su cosa si intende per "violazione contestata".

A chiarirlo, il recente Lodo pronunciato dal Collegio di Garanzia n. 1/2021 che vale la pena ripercorrere nel percorso motivazionale. In quel caso, un agente sportivo, in qualità di parte istante, adiva il Collegio di Garanzia arbitrale, chiedendo il pagamento di una somma a titolo di retribuzione da parte del suo assistito e in virtù del contratto di mandato da questi sottoscritto. Tra le varie eccezioni, parte intimata sollevava la decadenza di parte istante dall'esercizio dell'azione giudiziale facendo leva sull'art. 3, comma 2, Regolamento CONI agenti sportivi. Il Collegio arbitrale accoglieva l'eccezione di decadenza, evidenziando che il legislatore sportivo ha ancorato il dies a quo alla "violazione contestata" e non già alla "contestazione della violazione", e da ciò deducendo che, come già affermato in un precedente Lodo altro Collegio arbitrale, ${ }^{23}$ "laddove la violazione contestata si compendi nell'inadempimento di una obbligazione pecuniaria, il principio della mora ex re che presidia questo genere di obbligazioni (art. 1219, comma 2, n. 3) impone di ancorare il dies a quo all'atto della scadenza della pertinente obbligazione che, dovendo essere eseguita presso il domicilio del creditore, non necessita di apposita (ed autonoma) contestazione, diffida o messa in mora". In altre parole, secondo il Collegio, il termine decorre dal momento in cui si realizza l'inadempimento in occasione del mancato pagamento dell'obbligazione pecuniaria per inutile decorso del termine convenuto tra le parti. Nella fattispecie, dal momento che il contratto di mandato intercorso tra le parti prevedeva espressamente quale termine per l'adempimento dell'obbligazione pecuniaria il giorno 30 giugno 2020, ed essendo stata la domanda di arbitrato invece presentata il 2 settembre 2020, anche applicando il termine di sospensione feriale, risultava inutilmente decorso il termine decadenziale di venti giorni per l'instaurazione della procedura arbitrale.

È, dunque, il termine convenuto per l'adempimento del contratto il momento in cui la violazione si consuma, il dies a quo corretto da prendere a riferimento per l'introduzione della domanda di arbitrato, senza che a nulla rilevi qualsivoglia altra (e successiva) attività di contestazione.

Nonostante la soluzione del Collegio applichi norme di stretto diritto e non equità - come vorrebbe questo tipo di procedura - la tesi avanzata dal Collegio pare condivisibile. A fronte del fatto che, seppure con termine

${ }^{22}$ Così E. Zucconi Galli Fonseca, Diritto dell'arbitrato, Bologna, Bononia University Press, 2016, p. 486.

${ }^{23}$ Si trattava del Lodo arbitrale n. 6/2020 del Collegio di Garanzia dello Sport CONI che stabiliva in motivazione: "In disparte il problema se la pertinente eccezione, laddove tempestivamente e ritualmente spiccata dalla parte intimata, sia di decadenza (con connessa rilevabilità d'ufficio) ovvero non lo sia (configurando come tale una eccezione in senso proprio, rimessa esclusivamente alla parte interessata), interessa qui il profilo concernente il dies a quo assegnato alla parte istante dal menzionato art. 3, comma 2, del Regolamento per introdurre, esplicitamente a pena di decadenza, la procedura arbitrale. La norma di che trattasi testualmente recita: "la procedura arbitrale è introdotta, entro il termine perentorio di venti giorni dalla violazione contestata, con istanza rivolta al Collegio di Garanzia...", il pertinente dies a quo venendo dunque ancorato dal Legislatore sportivo alla "violazione contestata", e non già alla "contestazione della violazione". Ne consegue che, laddove la "violazione contestata" si compendi - come appunto nel caso di specie e in molti altri - nell'inadempimento ad una obbligazione pecuniaria, il principio della c.d. mora ex re che presidia questo genere di obbligazioni (art. 1219 comma 2, n. 3 c.c.) impone di ancorare il dies a quo di che trattasi al momento in cui, per dirla in gergo penalistico, la violazione "si consuma" e, dunque, all'atto della scadenza della pertinente obbligazione che, dovendo essere eseguita presso il domicilio del creditore, non necessita di apposita (ed autonoma) "contestazione", diffida o messa in mora". Nel caso di specie, complice la sospensione dei termini avutasi nel periodico pandemico del 2020, l'introduzione della pertinente domanda di arbitrato è stata comunque ritenuta tempestiva. 
improprio di "violazione", è all'inadempimento contrattuale che la norma regolamentare vuole riferirsi. Come noto, di fronte alla scadenza del debito e al perdurare della possibilità della prestazione, il ritardo nel soddisfacimento del creditore può essere qualificato come inadempimento soltanto ove sia realizzata la fattispecie del comma $1^{\circ}$ o sussistano i presupposti della mora ex re del comma $2^{\circ}$ dell'art. 1219 c.c. Nel caso in cui - invero il più frequente per tali tipologie di liti - la causa abbia ad oggetto l'accertamento dell'inadempimento contrattuale e dunque riguardi un'obbligazione pecuniaria, con previsione di un termine per il pagamento previsto dalle parti, dovrà applicarsi il principio della c.d. mora ex re che presidia questo genere di obbligazioni (art. 1219 comma 2, n. 3 c.c.), in quanto, alla scadenza, il pagamento della pertinente obbligazione deve essere eseguita presso il domicilio del creditore, non necessitando a tale proposito di apposita (ed autonoma) "contestazione" del credito o diffida o messa in mora.

Rimane, infine, da evidenziare che tale eccezione di decadenza dovrà essere sollevata dalle parti, essendo precluso un rilievo d'ufficio da parte dell'arbitro, in forza dell'art. 2969 c.c. ${ }^{24}$

\section{Considerazioni conclusive}

In conclusione, nel contesto sportivo, che da tempo conosce il fenomeno arbitrale ${ }^{25}$, rappresenta elemento di grande novità l'avvio innanzi al Collegio di Garanzia dello Sport - per effetto della recente emanazione del Regolamento CONI in materia - di procedure arbitrali atte a dirimere controversie nascenti in dipendenza dei contratti di mandato tra agenti sportivi e loro assistiti.

Non sono mancati però, fin dalle più recenti applicazioni dell'istituto, dubbi interpretativi in ordine alla sua disciplina e, tra i tanti, quello relativo al dies a quo per la proposizione della domanda arbitrale innanzi al Collegio di Garanzia, risolto nell'attenta individuazione del momento in cui si consuma la "violazione contestata".

Le criticità che vengono in rilievo sono figlie di un istituto di recentissima introduzione che ha trovato per il momento ancora timidi consensi, ${ }^{26}$ ma che non mancherà, ciononostante, di trovare sempre maggiore applicazione.

Rimane però sullo sfondo il problema del delicato regime applicativo dell'istituto, legato ad una concezione che vuole tradizionalmente l'arbitrato sportivo, come quello degli agenti di cui ci siamo occupati, come arbitrato irrituale $^{27}$, un istituto invero quasi totalmente scomparso nel campo della giustizia nell'ordinamento statale. Sono dell'idea che, a breve, bisognerà ripensare lo stampo irrituale di tali arbitrati, soprattutto in materie che coinvolgono interessi economici, anche a fronte delle sempre maggiori tutele richieste dagli operatori di un mondo, quello sportivo, sempre più complesso e sempre più oggetto di attenta regolamentazione normativa.

\footnotetext{
${ }^{24}$ Sul punto, anche il Lodo n. 3 del 2020 del Collegio di Garanzia secondo cui tale eccezione di decadenza "ai sensi dell'art. 2969 c.c., non può essere rilevata d'ufficio e che, pertanto, in mancanza di una valida formulazione di essa su istanza di parte, non può trovare accesso nel presente procedimento di arbitrato. Osserva al riguardo il Collegio che la rilevabilità ex officio di tale eccezione riguarda, a mente del citato art. 2969 c.c., esclusivamente i c.d. diritti indisponibili, il cui ambito rimane circoscritto ai diritti della personalità, come riconosciuti dalla Costituzione, ed agli status familiari, oltre che ad alcuni diritti patrimoniali di natura inviolabile come, ad esempio, il diritto alla retribuzione o alle ferie ovvero agli alimenti. Si tratta, pertanto, di diritti la cui natura ne impedisce la negoziabilità, trattandosi di profili che soddisfano, unitamente al titolare, anche interessi di natura pubblicistica".

${ }^{25}$ Il riferimento è, innanzitutto, all'arbitrato del lavoro sportivo introdotto ad opera dell'art. 4 della legge 23 marzo 1981, n. 91 . Trattasi di un insieme di collegi arbitrali esterni rispetto alla giustizia statale, comunque parte integrante del sistema sportivo in senso lato e concernente le liti patrimoniali che investono il campo dei diritti dei lavoratori rilevanti per la sfera giuridica statale. V. E. Zucconi Galli Fonseca, L'arbitrato nello sport: una better alternative, in Riv. dir. sportivo, 2016, p. 281 ss.; E. Fanesi, L'arbitrato quale strumento per una rinnovata autonomia dell'ordinamento sportivo, in Rivista di diritto ed economia dello sport, 2020 , p. 85 ss.; C. Rasia, L'arbitrato del lavoro nel calcio, in questa rivista, 3-4, 2017, p. 241 ss.

${ }^{26}$ Nei due anni di vita si registrano circa una quindicina di lodi. Si tratta dunque di un'area di contenzioso ancora circoscritta.

27 È ancora prevalente, in dottrina, l'idea che ritiene che la natura irrituale dell'arbitrato sportivo sia più funzionale alle esigenze dell'ordinamento sportivo, in considerazione della libertà delle forme e della maggiore stabilità della decisione che, per quanto priva di valenza di titolo esecutivo, è immediatamente esecutiva all'interno dell'ordinamento sportivo, in forza delle previsioni federali che introducono l'applicazione di sanzioni disciplinari a carico della parte soccombente che non esegue il lodo (cfr. E. Zucconi Galli Fonseca, Arbitrato nello sport: una better alternative, in www.coni.it, 2016).
} 